\title{
Rapid Differentiation From Human Induced Pluripotent Stem Cells Into Functional Oligodendrocytes Using Synthetic Modified Olig2 mRNA
}

\author{
Jian Xu \\ Sun Yat-sen University
}

\section{Rui Wang}

First People's Hospital of Foshan

Jingxin Zhao

Sun Yat-sen University

Fumei He

Sun Yat-sen University

\section{Yidi Zhang}

Sun Yat-Sen University

\section{Yuan Xie}

Sun Yat-sen University

\section{Lan Shen}

Shenzhen Hospital of Southern Medical University

\section{Yichu Nie}

Sun Yat-Sen University

\section{Wenbin Deng ( $\nabla$ dengwb5@mail.sysu.edu.cn )}

Sun Yat-Sen University https://orcid.org/0000-0003-2552-2553

\section{Research Article}

Keywords: Olig2, synthetic modified messenger RNA (smRNA), human pluripotent stem cells (hiPSCs), oligodendrocytes, cell transplantation

Posted Date: January 3rd, 2022

DOI: https://doi.org/10.21203/rs.3.rs-1192447/v1

License: (9) This work is licensed under a Creative Commons Attribution 4.0 International License. Read Full License 


\section{Abstract}

Background: Transcription factors (TFs) have been introduced to drive highly efficient differentiation of human induced pluripotent stem cells (hiPSCs) into lineage-specific oligodendrocytes (OLs). However, effective strategies currently rely mainly on genome-integrating viruses. To facilitate the translation of hiPSC-derived OLs into clinical practice, a synthetic modified messenger RNA (smRNA) reprogramming method that generates transgene-free OLs has been developed.

Methods: An smRNA encoding Olig2, a key TF in OL development, with a defined phosphorylation site modification serine 147 replaced with alanine, Olig2 ${ }^{\text {S147A }}$, was designed to reprogram hiPSCs into OLs. Proteomics were used to identify Olig2 ${ }^{\mathrm{S147A}}$-binding proteins that positively mediated of Olig2 ${ }^{\mathrm{S} 147 \mathrm{~A}}$-driven OL differentiation.

Results: We demonstrated that repeated administration of the smRNA encoding Olig2 ${ }^{\text {S147A }}$ led to higher and more stable protein expression. Using the single-mutant Olig2 smRNA with morphogens, we established a 6-day smRNA transfection protocol, and glial induction led to rapid NG2 ${ }^{+}$OL progenitor cell (OPC) generation ( $>70 \%$ purity) from hiPSC-derived neural progenitor cells (NPCs). The smRNA-induced $\mathrm{NG}^{+}$OPCs matured into functional OLs and myelinated nanofibers in vitro. Moreover, when transplanted into mice with cuprizone-induced demyelination, smRNA-induced OPCs promoted remyelination ex vivo. A proteomic analysis of Olig2-binding proteins indicated that the heat shock protein 70 (HSP70) complex bound Olig2. The HSP70 complex bound more strongly to Olig2 with the modified phosphorylation site than to wild type Olig2. VER-155008, an HSP70 complex antagonist, and ML346, an HSP70 complex agonist, inhibited and promoted Olig2 transcriptional activity and efficient OL generation, respectively.

Conclusions: We present a very safe and efficient smRNA-driven strategy for hiPSC differentiation into OLs, which might be utilized for disease modeling, drug discovery, and/or therapeutic OPC/OL transplantation in neurodegenerative disease.

\section{Introduction}

Oligodendrocytes (OLs), myelinating cells in the central nervous system (CNS), are extremely promising therapeutic targets in cell replacement-based therapy for myelin loss or dysfunction, such as that in multiple sclerosis and white matter ischemic injury (1-4). Reprogramming human induced pluripotent stem cells (hiPSCs) to OLs is a profoundly promising approach to disease modeling, drug development, and potentially therapeutic $\mathrm{OL}$ transplantation. Research advances have made it possible to generate surface antigen 04 (04)-positive and $\mathrm{MBP}^{+}$OLs from hiPSCs within only 20 days by overexpressing key transcription factors (TFs) (5). However, the traditional approach to achieving TF overexpression is generally based on ectopic viral-mediated gene delivery, and viral integration into the genome initially is a formidable obstacle to the therapeutic use of OLs. To this end, synthetic modified messenger RNA (smRNA) in vitro has been developed to diminish the innate immune response and stabilize delivery of genetic material into mammalian cells that can be efficiently translated into specific functional 
proteins (6-8). In contrast to DNA-based gene manipulation, the introduction of smRNA carries no risk of genomic integration, and smRNA can be translated in the cytoplasm without delivery into the nucleus, which means that smRNAs results in a safer and more efficient method for inducing protein expression.

Instability and a small window for protein expression are the major obstacles to using smRNA for cell reprogramming. For mRNA to be effectively translated in vitro, the 5'-terminal m7GpppG cap and the 3'terminal poly $(A)$ sequence are incorporated into mRNA structure for in vitro transcription. 5-Methyl-cTP, pseudo/ $\psi$-UTP and other modified nucleotides can also be incorporated into mRNA to reduce immunogenicity and enhance stability (9). smRNA can be used to directly reprogram the fate of human cells into iPSCs (10). Warren et al. employed smRNA to drive the expression of "reprogramming" factors and successfully developed a method for hiPSC generation (11). In particular, this method of protein expression mediated by modified RNA has the potential to become a very useful technology for cellbased therapies and regenerative medicine. Moreover, modified RNA can be used to direct the fate of reprogrammed hiPSCs into tissue-specific cell types $(12,13)$; however, mRNA-driven differentiation of OLs derived from hiPSCs has been largely unreported. We and others have sought to develop protocols to achieve uniform and reproducible culturing of hiPSC-derived OL progenitor cells (OPCs) $(5,14-18)$. We identified factors to facilitate the differentiation of many OPCs derived from hiPSCs, optimized methods and defined conditions, and tested the survivability and differentiation of the cells in animal models. Transplantation of iPSC-derived OPCs that have been better "instructed" to follow the OL lineage may facilitate recovery of patients with CNS diseases $(14,19)$. The regulation of TF Olig2 expression affects a major regulatory transcription pathway in $\operatorname{OL}$ genesis $(20,21)$. Overexpressing Olig2 in primary neural stem cells (NSCs) has been reported to facilitate myelinating OL generation and contribute to remyelination of the corpus callosum in mice with experimental allergic encephalomyelitis (EAE) (22). Although previous studies have shown that oligodendroglial lineage marker genes, such as nerve/glial antigen 2 (NG2, also known as CSPG4) or platelet-derived growth factor receptor alpha (PDGFRa, also known as CD140a), were not induced following only TF Olig2 overexpression in neural progenitor cells (NPCs) (17), our unpublished data show dysfunction in regulating motor neuron (MN) development and improved OL specification by regulating posttranslational modifications of the Olig2 protein. Therefore, it seems that the significance of Olig2 overexpression in OL specification should be further emphasized. Indeed, posttranslational modifications of Olig2 proteins have been shown to influence cell fate transitions. Li et al. reported that specific serine sites (147) in Olig2 are essential for MN-OL switching. Replacing serine 147 with an alanine residue (S147A) abolished MN production without preventing OL formation (23). We expect that we will establish better OPC differentiation protocols from hiPSCs by forced expression of the smRNA coding mutant Olig2 (Olig2 ${ }^{\text {S147A }}$ ), and single Olig2 smRNA may be used in more efficient protocols of mRNA-driven OL differentiation from hiPSCs.

In this study, we established novel repeated administration of smRNA for efficient and rapid hiPSC differentiation into OL lineage-specific cells and found that phosphosite modification of Olig2 can effectively promote the efficiency of this smRNA-driven hiPSC differentiation process. Therefore, to the 
best of our knowledge, the first single viral-free smRNA-driven method will widen the application of OL replacement therapy in various diseases involving myelin injury.

\section{Methods}

\section{mRNA Synthesis and Transfection}

Coding sequences of human Olig2 ${ }^{\mathrm{WT}}$ and Olig2 ${ }^{\text {S147A }}$ were cloned into a vector containing the T7 promoter and poly (A) tail for in vitro transcription. Open reading frames (ORFs) of Olig2 were cloned into the PCR2-UTR-R1R2 vector following the LR reaction protocol (Thermo Fisher Scientific, USA). PCR2-UTR$B 1 B 2$ vector was linearized with a restriction enzyme that cuts outside the ORF. The linearized vector was used as the template in a tail PCR reaction which utilized a 5' primer with a T7 polymerase promoter sequence and a 3' UTR directed primer with a long poly $T$ tail. The PCR product was gel purified and was used as a template in an in vitro transcription (IVT) protocol essentially as described by Mandal and Rossi. The IVT reaction included an anti-reverse cap analog (ARCA) and modified nucleotides, 5-methyl cytidine-5'-triphosphate and pseudouridine-5'-triphosphate, to reduce cytotoxicity due to the activation of innate immune responses. The purified synthetic mRNA obtained was size verified and stored as aliquots at $-80^{\circ} \mathrm{C}$ until use. For each well of the 12-well plate, we incubated $0.2 \mu \mathrm{g}$ mRNA with $1.5 \mu \mathrm{L}$ Screenfect transfection reagent (299-73203, Wako, Japan) in $75 \mu \mathrm{L}$ transfection buffer for 15 minutes before adding to cell cultures. Cell proliferation and death analysis use the MTT (B7777, APExBio, Houston, TX) kit, following the manufacturer's instruction.

\section{hiPSC culture and neural differentiation}

For growth under feeder-free conditions, hiPSCs (kindly provided by Dr. Jiekai Chen, Guangzhou Institutes of Biomedicine and Health Chinese Academy of Science, China) were cultured on Matrigel-coated 12-well plates, using mTeSR (Stem Cell Technologies, Canada) medium. Cells were incubated at $37^{\circ} \mathrm{C}$ with $5 \%$ $\mathrm{CO}_{2}$, and were mechanically split every 5 days at 1:8 splite ratios using ReLeSR (Stem Cell Technologies, Canada) as described by manufacturers.

Differentiation into NPCs from hiPSC as previously described $(14,24)$. Briefly, hiPSC were collected from the 20th-30th passage, and were dissociated enzymatically and mechanically. The diluted colonies were replated into a six-well plate and added neural induction medium 1 (NIM1) for 2 days. The cultured medium was then switched to neural induction medium 2 (NIM2) for another 5 days. The cultures were then split onto matrigel-coated plates and cultured in neural progenitor cells maintenance Medium (NSMM) supplemented with Y-27632 (10 $\mu \mathrm{M}$, Med Chem Express, USA). For the NIM1, NIM2, and NSMM detail compositions used, see supplementary table S1. All experiments involving human stem cells were performed with the approval of the Sun Yat-sen University Medicine Institutional Review Boards (SYSUIACUC-2021-000776).

\section{OPC differentiation}


For oligodendrocytes lineage differentiation, a two-step differentiation protocol was utilized. hiPSCderived NPCs were seeded at $1.5 \times 10^{5}$ cells per well in the 12 well-plate coated with poly-Lornithine/laminin (Sigma-Aldrich, USA). NPCs were transfected with Olig2 WT or Olig2 S147A mRNA by ScreenFect transfection reagent for 6 days, and the culture medium was changed to glial induction medium (GIM) daily. For the GIM, and DM detail compositions used, see supplementary table S4. Cells can also be cryopreserved in medium containing 40\% neurobasal medium with B27 supplement, $50 \%$ fetal bovine serum and 10\% DMSO.

\section{Immunofluorescence staining and immunohistochemically staining}

Cells fixed with $4 \%$ paraformaldehyde for $15 \mathrm{~min}$ at room temperature and brain sections were processed for immunofluorescence staining. After fixation, the coverslips were washed with PBS three times and then blocked with $4 \%$ bovine serum albumin (BSA) for $30 \mathrm{~min}$ at $37^{\circ} \mathrm{C}$ and then incubated the corresponding primary antibody for overnight. On the next day, the coverslips were incubated in fluorescence-conjugated secondary antibodies (Invitrogen, San Diego, CA) for $1 \mathrm{~h}$ at room temperature in the dark. Coverslips were washed three time with PBS, stained with 4',6-diamidino-2-phenylindole (Vector Laboratories, Bulingame, CA) at least 10 min, then washes with PBS three times, and mounted with mounting medium (Invitrogen, San Diego, CA). Cells on the coverslips were imaged by using a fluorescence microscope (Eclipse Ti2-U, Nikon, Japan). All related primary antibodies in this article were given in supplementary table S2. For luxol fast blue (LFB, G1030, Servicebio, China) staining, Paraffinembedded coronal sections of brains were stained with LFB for examination of demyelination.

\section{In vitro myelination assay}

To assess the in vitro myelination capacity of smRNA-induced OPCs, $\mathrm{O}^{+}$cells were purified at differentiation day 21 by magnetic cell sorting (MACS) using anti-04 MicroBeads (Miltenyi Biotec) following the manufacturer's protocol. $04^{+}$OPCs were reseeded at a density of $5 \times 10^{4}$ cells per chamber on the $675 \mathrm{~nm}$ nanofiber chamber slides (Nanofiber Solutions, Sigma, USA) were precoated with 10 $\mu \mathrm{g} / \mathrm{mL}$ laminin (Sigma) and incubated with DM supplemented with $2 \mathrm{ng} / \mathrm{mL}$ BDNF and $2 \mathrm{ng} / \mathrm{mL}$ GDNF at $37^{\circ} \mathrm{C}$ overnight. Half of the medium was changed every other day and cells were fixed in $4 \%$ PFA after 14 $\mathrm{d}$ for Immunofluorescence staining.

\section{Immunoblotting,}

Whole cell lysates or nuclear protein lysate were extracted and then concentrated with BCA protein assay kit (Thermo Fisher Scientific, Waltham, MA). 50 $\mathrm{g}$ of the total protein were loaded into each well and separated by $10 \%$ SDS-PAGE electrophoresis. The primary antibodies are listed in Supporting Information Table S1. Secondary antibodies were labeled with HRP, and proteins were normalized to the housekeeping protein tubulin protein in the same blotted sample. 


\section{Immunoprecipitation (IP)}

Twenty-four hours after mRNA transfection, whole cell lysates were extracted and then concentrated with BCA protein assay kit (Thermo Fisher Scientific, Waltham, MA). $10 \mu \mathrm{g}$ of primary antibodies or isotype control IgG were covalently cross-linked to $25 \mu \mathrm{L}$ of protein $\mathrm{A} / \mathrm{G}$ magnetic beads (Thermo Fisher). A portion of each sample was saved as input. An equal amount $(1 \mathrm{mg})$ of each protein extract was incubated with protein A/G magnetic beads cross-linked with anti-Flag antibody (AP1013A, abcepta, USA) or isotype control IgG (3900s, cell signaling technology, USA) overnight at $4^{\circ} \mathrm{C}$. Pierce Protein $\mathrm{A} / \mathrm{G}$ Magnetic Beads were washed three times using Pierce IP Lysis/Wash Buffer for LC-MS/MS analysis.

\section{LC-MS/MS}

Aliquots of proteins were mixed with $200 \mu \mathrm{L}$ of $8 \mathrm{M}$ urea in Nanosep Centrifugal Devices (PALL). The device was centrifuged at $12,000 \mathrm{~g}$ at $20^{\circ} \mathrm{C}$ for $20 \mathrm{~min}$. All following centrifugation steps were performed applying the same conditions allowing maximal concentration. Then, $200 \mu \mathrm{L}$ of $8 \mathrm{M}$ urea solution with 10 mM DTT were added, and the reduction reaction was kept for $2 \mathrm{~h}$ at $37^{\circ} \mathrm{C}$. The solution was removed by centrifugation, and $200 \mu \mathrm{L} 8 \mathrm{M}$ urea solution with $50 \mathrm{mM}$ iodoacetamide (IAA) was added. The sample was incubated in the dark for $15 \mathrm{~min}$ at room temperature. The ultra-fraction tube was washed with 200 $\mu \mathrm{l}$ of $8 \mathrm{M}$ urea three times and $200 \mu \mathrm{L}$ of $25 \mathrm{mM}$ ammonium bicarbonate three times by centrifugation at $12,000 \mathrm{~g}$ for $20 \mathrm{~min}$ at room temperature. Then, $100 \mu \mathrm{L}$ of $25 \mathrm{mM}$ ammonium bicarbonate containing $0.01 \mu \mathrm{g} / \mu \mathrm{L}$ trypsin was added to each filter tube. The tubes were incubated at $37^{\circ} \mathrm{C}$ for $12 \mathrm{~h}$. The filter tubes were washed twice with $100 \mu \mathrm{L}$ of $25 \mathrm{mM}$ ammonium bicarbonate by centrifugationat $12,000 \mathrm{~g}$ for $10 \mathrm{~min}$. The flow-through fractions were collected and lyophilized. The lyophilized peptide fractions were re-suspended in $\mathrm{ddH}_{2} \mathrm{O}$ containing $0.1 \%$ formic acid, and $2 \mu \mathrm{L}$ aliquots of which was loaded into a nanoViper C18 (Acclaim PepMap 100,75 $\mathrm{mm} \times 2 \mathrm{~cm}$ ) trap column. The online Chromatography seperation was performed on the Easy nLC 1200 system (ThermoFisher). The trapping, desalting procedure were carried out with a volumn of $20 \mu \mathrm{L} 100 \%$ solvent A ( $0.1 \%$ formic acid). Then, an elution gradient of $5-38 \%$ solvent $B$ ( $80 \%$ acetonitrile, $0.1 \%$ formic acid) in 120 min was used on an analytical column (Acclaim PepMap RSLC, $75 \mu \mathrm{m} \times 25 \mathrm{~cm}$ C18-2 $\mu \mathrm{m} 100 \AA$ A). DDA (data-dependent acquisition) mass spectrum techniques were used to acquire tandem MS data on a Thermo Fisher Q Exactive mass spectrometer (Thermo Fisher, USA) fitted with a Nano Flex ion source. Data was acquired using an ion spray voltage of $1.9 \mathrm{kV}$, and an interface heater temperature of $275^{\circ} \mathrm{C}$. For a full mass spectrometry survey scan, the target value was $3 \times 10^{6}$ and the scan ranged from 350 to $2,000 \mathrm{~m} / \mathrm{z}$ at a resolution of 70,000 and a maximum injection time of $100 \mathrm{~ms}$. For the MS2 scan, only spectra with a charge state of 2-5 were selected for fragmentation by higher-energy collision dissociation with a normalized collision energy of 28 . The MS2 spectra were acquired in the ion trap in rapid mode with an AGC target of 8,000 and a maximum injection time of $50 \mathrm{~ms}$. Dynamic exclusion was set for $25 \mathrm{~s}$. The MS/MS data were analyzed for protein identification and quantification using PEAKS Studio 8.5. The local false discovery rate at PSM was $1.0 \%$ after searching against target database with a maximum of two missed cleavages. The following settings were selected: Oxidation (M), Acetylation (Protein N-term), Deamidation (NQ), Pyro-glu from E, Pyro-glu from $\mathrm{Q}$ for variable modifications as well as fixed Carbamidomethylation of cysteine. Precursor 
and fragment mass tolerance were set to $10 \mathrm{ppm}$ and $0.05 \mathrm{Da}$. The Label free quantitative parameter is set as: fold change $1.2 \bigotimes$ respectively.

\section{Flow cytometry analysis}

Cells were enzymatically harvested using accutase, centrifuged and resuspended in $100 \mu \mathrm{L}$ FACS buffer (1X PBS, $2 \%$ fetal bovine serum and $0.02 \%$ sodium azide). To determined NG2, PDGFRa, and 04 expression, Olig2-induction progenies were incubated with 1/20 dilution of NG2-PE, PDGFRa-APC, and O4-APC antibody or unstained control for $15 \mathrm{~min}$ at $4^{\circ} \mathrm{C}$, washed and re-suspended in $150 \mu$ l FACS buffer. Cells were analyzed on BD flow cytometer. After exclusion of cell doublets, gates were established based on the corresponding unstained controls (Fig S4B-C). All flow cytometric data was further analyzed using the BD CytoExpert software (BD Biosciences, San Jose, CA).

\section{Transplantation of mRNA-induced OPCs in cuprizone-induced demyelination mouse model}

8-10-week female C57BL/ 6 mice $(n=5)$ were fed with AIN-93G purified rodent diet with $0.2 \%$ Cuprizone (Dyets, D200218) for 5 weeks, thereafter cuprizone-infused food was removed and the mice were given normal diet. The demyelination mice induced by cuprizone were serve a well proved model to test the functional capacity of transplanted OPCs toward forming MBP-positive compact myelin sheath around axons. The demyelination mice were injected with 100000 Olig2 ${ }^{\mathrm{S} 147 \mathrm{~A}} \mathrm{mRNA}$-induced $04^{+} \mathrm{OPCs}$ corpus callosum using a Hamilton syringe at the following stereotaxic coordinates: anteroposterior 0.2, mediolateral 1.1 with Bregma as a reference, and dorsoventral $2 \mathrm{~mm}$ from the skull surface according to our previously published protocols (19). Mice were administered cyclosporine $10 \mathrm{mg} / \mathrm{kg}$ daily intraperitoneally for immune suppression, beginning 2 days before cell transplantation until sacrifice. Postoperative care was given according to the Institutional Animal Care and Use Committee (IACUC) protocols approved by Sun Yat-sen University. Mice were sacrificed after 8 weeks, and the corpus callosum sections $(2-3 \mathrm{~mm})$ containing the injection site was microdissected and processed for imaging under an electron microscope or for confocal imaging.

\section{Transmission electron microscope}

Brains were isolated from 4\% PFA perfused mice, and then the corpus callosum were isolated and fixed with PBS containing $2.5 \%$ glutaraldehyde for 2 hours. Next, the corpus callosum were washed, fixed with $1 \%$ osmium tetroxide, dehydrated in acetone, and embedded in EPON. Afterwards, $70-80 \mathrm{~nm}$ thin sagittal sections cut with a diamond knife were mounted on copper slot grids coated with Formvar. After staining with uranyl acetate and lead citrate, they were scanned on JEM-1230 transmission electron microscope. Approximately 100 remyelinated axons were measured for each group, and G-ratios were calculated using Image Pro.

\section{Data Analysis and Statistics}


All experiments were performed at least three independent replications. Post hoc tests including the Student's $t$ test and the Tukey multiple-comparison texts were analyzed by Prism software (PRISM ${ }^{\text {TM }}$ 8.0.2) as appropriate. The data were represented as mean \pm standard error of the mean (SEM). Differences between mean values for different treatments were considered to be significant at $P<0.05$

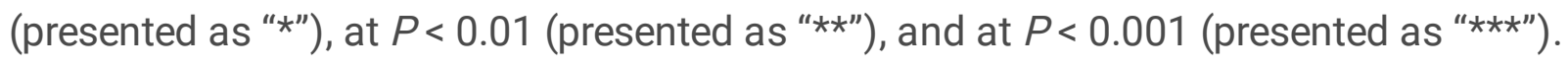

\section{Results}

\section{Synthetic mRNA coding Olig2 with phosphorylation site modification drives highly efficient oligodengroglial lineage cell conversion of hiPSC-derived NPCs}

We delivered smRNA to express a mutant Olig2 protein that recapitulates the induction of oligodengroglial TFs during OL development. Two vectors were constructed for T7 promoter-driven in vitro transcription to synthesize mRNAs coding wild-type Olig2 and mutant Olig2 with a specific serine-toalanine modification (Fig. 1A, Fig. S1). Before testing these Olig2 smRNAs, we first determined the efficiency of smRNA delivery into NPCs derived from hiPSCs. Using a cationic liposome-based delivery system, we introduced eGFP mRNA into NPCs as an in vitro tracer. As shown in Fig. 1B, single transfection of $300 \mathrm{ng}$ of eGFP mRNA into NPCs showed transfection efficiency greater than $95 \%$. Moreover, immunofluorescence staining for Flag led to the identification of Flag-tagged Olig2 ${ }^{\mathrm{WT}}$ and Flagtagged Olig2 ${ }^{\text {S147A }}$ expression in NPCs 24 hours posttransfection (Fig. 1C). These results indicated that these smRNAs were successfully delivered into NPCs and translated into proteins. Next, the possibility of smRNA-induced cytotoxicity, which would have decreased the efficiency of cell reprogramming, was determined by Cell Counting Kit-8 (CCK-8) assay, and compared to the effect in untransfected cells, no significant cytotoxicity was observed upon smRNA treatment (Fig. S2). Type I interferon (IFN) has been considered a marker for antiviral defense responses in mammalian cells through interferon- $\gamma$ - and NF-kbdependent pathways (25). The expression of IFN signaling markers was transient, as evidenced by significant downregulation 48 hours after mRNA transfection. These results supported our strategy of mutating Olig2 smRNA to drive NPC differentiation into OLs.

Blocking Olig2 phosphorylation at serine 147 has been shown to abolish MN production without preventing $\mathrm{OL}$ production and triggers the switch from an $\mathrm{MN}$ to an $\mathrm{OL}$ fate (23). Using our optimized strategy for smRNA synthesis and transfection, we tested smRNAs coding Olig2 in wild-type and phosphorylation site-modified forms to determine which protein induces greater protein expression and OL conversion. smRNA coding Olig2 with the S147A phosphorylation site modification resulted in 1.2-fold higher protein expression than wild-type Olig2, as quantified 16 hours posttransfection, and reached a peak 24 hours after smRNA transfection (Fig. 1D). We then asked whether Olig2 ${ }^{\text {WT }}$ and Olig2 ${ }^{\text {S147A }}$ smRNAs more potently induce their validated downstream targets, such as $50 X 10$, which is required for OL differentiation and maturation (26). As shown in Figure 1E, three or six daily transfections of smRNAs were performed to determine the most suitable and efficient differentiation strategy. NPCs derived from hiPSCs were plated in 12-well plates precoated with the reduced growth factor Matrigel/laminin for determining SOX10 mRNA expression. The quantitative polymerase chain reaction (qPCR) results 
suggested that six daily transfections of Olig2 ${ }^{\mathrm{S} 147 \mathrm{~A}}$ induced a 9.8-fold higher SOX10 expression level than three daily transfections. Interestingly, we found that Olig2 ${ }^{\mathrm{S147A}}$ smRNA drove higher OL lineage marker gene expression than Olig2 ${ }^{\mathrm{WT}}$ smRNA (Fig. 1F). Thus, the smRNA coding Olig2 with a phosphorylation site modification was the better driver of OL conversion from hiPSC-derived NPCs than their respective wild-type counterpart.

\section{hiPSC differentiation into smRNA-induced OPCs}

Since six daily smRNA transfections showed higher OL differentiation potential, we next established 6day mRNA transfection schemes for mRNA-induced OLs derived from hiPSCs (Fig. 2A). First, NANOGand SSEA4-positive hiPSCs (Fig. 2B-D) were differentiated into NPCs with neural induction medium (NIM) for 7 days, and the cultured cells expressed PAX6-and NESTIN (Fig. 2C-E). One day after mRNA transfection, the culture medium was replaced with glial induction medium (GIM) containing different morphogens (T3, SAG and PDGFRa). After 6 days, the GIM was changed to differentiation medium (DM) lacking PDGF and SAG to promote OL generation and maturation. Six days after mRNA treatment and GIM induction, smRNA-induced NG2+ OPCs were identified. In contrast, NPCs that had received eGFP mRNA failed to attach or survive in culture with GIM (data not shown); however, NPCs that received Olig2 ${ }^{\mathrm{S} 147 \mathrm{~A}}$ smRNA promoted $\mathrm{NG}^{+} / \mathrm{SOX} 10^{+} / \mathrm{PDGFRa}^{+}$OPC generation to a greater degree than NPCs that received Olig2 ${ }^{\mathrm{WT}}$ mRNA (Fig. 2F).

To further characterize the OPC generation induced by Olig2 ${ }^{\text {S147A }}$ smRNA, we analyzed the cultured cells after 6 days of transfection by flow cytometry. The smRNA-induced cultures largely comprised NG2 ${ }^{+}$cells (74.94 $\pm 2.68 \%)$, in contrast to the Olig2 ${ }^{\mathrm{WT}}$ mRNA-induced cultures $(27.72 \pm 1.25 \%$, Figure 2E-F). PDGFRa, another marker, was utilized for OPC identification (27). Accordingly, 32.82\% $\pm 2.68 \%$ PDGFRa ${ }^{+}$OPCs were observed in Olig2 ${ }^{\text {S147A }}$ smRNA-induced cultures; however, only $6.68 \pm 1.25 \%$ PDGFRa $^{+}$OPCs were observed in Olig2 ${ }^{\mathrm{WT}}$ smRNA-induced cultures (Fig. 2G-H). Blocking the phosphorylation of Olig2 downregulated the expression of the MN-specific marker NGN2, which resulted in the initiation of oligodendrocyte production (23). We then determined the NGN2 mRNA expression levels in Olig2 ${ }^{\mathrm{WT}}$ mRNA and Olig2 ${ }^{\text {S147A }}$ mRNA-induced cultures and found that Olig2 ${ }^{\text {S147A }}$ mRNA significantly downregulated NGN2 mRNA expression to a greater extent than Olig2 ${ }^{\mathrm{WT}} \mathrm{mRNA}$, but it upregulated $N K X 2.2$ expression, which was required for OPC/OL differentiation (Fig. 2l). Another study showed that the mRNA expression level of $H B$, a downstream TF of $N G N 2$, was also decreased in Olig2 ${ }^{\text {S147A }}$ mRNAinduced OPCs (Fig. 2I). Taken together, the smRNA coding Olig2 with phosphorylation site modification facilitated highly efficient OPC generation.

\section{Maturation of Olig2 smRNA-induced OPCs into myelinating OLs}

Differentiation of OPCs into mature OLs under both extrinsic and intrinsic factor stimulation is a key event for axonal myelination in the CNS. We next investigated whether smRNA-induced OPCs can further mature into functional OLs. The 04 epitope, which was a mature OPC or pre-OL marker, was utilized to 
determine the terminal differentiation of the OPCs (28). To determine the kinetics, efficiency and yield of mRNA-mediated OL lineage specification, we performed weekly flow cytometry assays to determine $04^{+}$ $\mathrm{OPC} / \mathrm{OL}$ generation in our culture system. As shown in Fig. 3A-B, Olig2 ${ }^{\mathrm{S147A}} \mathrm{mRNA}$-induced $04^{+}$cells initially measured at $40.49 \pm 2.54 \%$ on day 14 increased to $77.16 \pm 6.24 \%$ by day 21 ; however, only $0.09 \pm$ $1.25 \% 04^{+}$cells on day 7 and $11.97 \pm 2.24 \% 04^{+}$cells on day 21 were identified in the Olig2 ${ }^{\mathrm{WT}}$ mRNAtreated cultures. Expression of myelin basic protein (MBP), a membrane actin-binding protein in CNS myelin, is a critical measure of OLs and multilayered compact myelin (29). Immunofluorescence staining for MBP $f$ confirmed that Olig2 ${ }^{\text {S147A }}$ mRNA treatment led to rapid differentiation and maturation of OPCs compared with wild-type mRNA (Fig. 3C-D). In line with our previous data, on day 21 of differentiation, OLs after Olig2 ${ }^{\text {S147A }}$ mRNA induction showed upregulated levels of MBP and MOG, by 3.4-fold $(P<0.001)$ and 2.6-fold $(P<0.003)$, respectively, compared to those in the Olig2 ${ }^{W T}$ mRNA-induced OLs (qPCR assay, $n$ $=3$, Fig. $3 E$ ). This outcome was associated with a concurrent increase in the expression of other myelin genes, i.e., myelin-associated glycoprotein (MAG) by 3.5-fold ( $p$ value $=0.005, n=3$ ) and proteolipid protein 1 (PLP1) by 3.2-fold ( $P$ value $=0.039, n=3$, Fig. 3E). Together, these results indicated that Olig2 ${ }^{W T}$ mRNA-induced OPCs showed limited maturation, whereas Olig2 ${ }^{\text {S147A }}$ smRNA-induced OPCs were capable of maturing toward the terminal stage because critical myelin genes were highly expressed.

To assess the myelination properties of the OLs in vitro, we performed a coculture study with electrospun nanofibers. When FACS-purified $\mathrm{O4}^{+}$cells were plated on either a laminin-coated surface (Fig. 3F) or plates containing 675-nm electrospun nanofibers for 3 weeks, they continued to differentiate into mature $\mathrm{MBP}^{+} \mathrm{OLs}$ (Fig. 3G). The cocultures were stained with an antibody against MBP, and $\mathrm{MBP}^{+}$cells displayed the distinct, typical branched morphology of mature OLs. The cells cultured on the electrospun nanofibers developed processes that were aligned along the nanofiber and appeared to myelinate the fibers (Fig. $3 \mathrm{H}$ ). Taken together, these results suggested that treatment with Olig2 ${ }^{\mathrm{S147A}}$ smRNA increased OPC differentiation into $\mathrm{MBP}^{+}$functional OLs and that Olig2 ${ }^{\mathrm{S147A}}$ mRNA-OLs possessed myelinating properties in vitro.

\section{Olig2 smRNA-induced OLs generated a multilayer myelin sheath in mouse brains with cuprizone-induced demyelination}

To address myelination in an in vivo context, FACS-purified Olig2 ${ }^{\text {S147A }}$ (Fig. S4) smRNA-induced $04^{+}$cells were injected into the corpus callosum of mouse brain with cuprizone-induced demyelination, and mouse brain slices were obtained 8 weeks later (Fig. 4A-B). Then, immunohistochemistry demonstrated efficient engraftment, which was identified by the presence of human $\mathrm{hN}^{+}$cells with coexpression of MBP in brain tissue (Fig. 4C). We next sought to investigate the formation of compact myelin ultrastructure around mouse axons in vivo by Olig2 ${ }^{\text {S147A }}$ smRNA-induced OLs. Transition electron microscopy was performed with samples of the corpus callosum derived from mice with cuprizone-induced demyelination that had received vehicle or Olig2 ${ }^{\mathrm{S} 147 \mathrm{~A}}$ smRNA-induced $04^{+}$OPCs $(n=5)$. We found that the corpus callosum in the cell-transplanted mice was densely myelinated by mature compact myelin, which was characterized by major concentrically organized dense lines (Fig. 4D-E) and interlaminar tight junctions. The density of the 
myelinated axons in the OPC group was significantly higher than that in the vehicle group (Fig. 4F-G). The remyelination by engrafted OPCs was further analyzed and quantified by g-ratio, which is the ratio of the axon diameter to the total diameter of a myelinated fiber (30). Compared to the vehicle group, the OPC group showed significantly lower g-ratio values (Fig. 4H). Luxol fast blue (LFB) staining showed spontaneous remyelination in the OPC group (Fig. 4I). These results indicated that Olig2 smRNA-induced OLs matured into myelin-forming cells and enhanced the remyelination process in the demyelinated mouse brain.

\section{The HSP70 complex binds to and promotes the function of Olig2 in driving OL differentiation}

To confirm the underlying mechanism of OL differentiation mediated by Olig2 smRNA and determine the potential partners that promote $\mathrm{OPC} / \mathrm{OL}$ generation, we performed proteomic analysis to identify

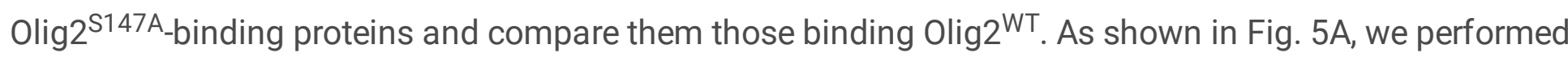
Flag IP to pull down Flag-tagged Olig2 ${ }^{\mathrm{WT}}$ and Olig2 ${ }^{\mathrm{S} 147 \mathrm{~A}}$ and their binding proteins from NPCs following 24 hours of transfection with either Olig2 ${ }^{\mathrm{WT}}$ and Olig2 ${ }^{\text {S147A }}{ }^{\mathrm{mRNA}}$, respectively (Fig. 5B). The proteomic analysis led to the identification of 67 differentially expressed proteins in NPCs transfected with either Olig2 ${ }^{\text {WT }}$ or Olig2 ${ }^{\text {S147A }}$ mRNAs (Supplemental table S1). Interestingly, previous studies performed in our laboratory revealed poly (ADP-ribosyl) polymerase-1 (PARP-1) is an intrinsic driver of OL development and myelination by stabilizing myelin gene mRNA translation. Specific inhibition of PARP1-mediated PARylation activity in Olig2-expressing cells further compromised the OL differentiation and CNS remyelination (31). Indeed, in line with our proteomic data, PARP-1 expression associated with Olig2 $2147 \mathrm{~A}$ was $\sim 5$-fold higher than that associated with Olig2 ${ }^{\text {WT }}$. This suggested that PARP- 1 might be a crucial factor that mediates Olig2 posttranslational modification involved in OL differentiation, and increased PARP-1 binding to Olig2 was consistent with enhanced protein stability and elevated myelin protein expression, which were shown in Figure 1.

These differentially binding proteins were subjected to pathway enrichment analysis in the DAVID bioinformatics database (Supplemental table S2). The myelin sheath was identified (Fig. 5C) and might be a positive modulator of Olig2 transcriptional activity that drives more efficient OL differentiation. Among these elevated proteins enriched in the myelin sheath, proteins associated with Olig2 ${ }^{\text {S147A }}$ were found to include multiple chaperones, particularly three main members (HSPA5, HSPA8, and HSPA9) of the heat shock protein 70 (HSP70) complex (Fig. 5D), indicating a potential role for the HSP70 complex in regulating Olig2-driven $\mathrm{OL}$ differentiation.

To validate the identity of the TF Olig2-binding proteins in the nuclear fraction, nuclear proteins were extracted from NPCs 24 hours after transfection of either Olig2 WT or Olig2 ${ }^{\text {S147A }}$ mRNAs. Immunoprecipitation (IP) and immunoblot analysis were performed to validate the two candidate Olig2binding nuclear proteins, HSPA8 and HSPA9, which are mainly located in the nucleus and cytosol (32). Indeed, based on our working model (Fig. 6A), HSPA8 and HSPA9 were consistently more highly associated with Olig2 ${ }^{\mathrm{S} 147 \mathrm{~A}}$ than with Olig2 ${ }^{\mathrm{WT}}$ (Fig. 6B). Next, the HSP70 complex agonist ML346 (ML) 
and antagonist VER-1555008 (VE) were utilized to determine whether the efficiency of OL generation was regulated by HSP70 activity. ML346 is an activator of HSP70 expression and HSF-1 activity (33). ML treatment promoted the yield of MBP-positive OLs by $45 \%$ following Olig2 ${ }^{\text {S147A }}$ mRNA induction (Fig. 6CD), suggesting that the HSP70 complex is a positive modulator of Olig2 ${ }^{\mathrm{S} 147 \mathrm{~A}}$ activity. In contrast, VER is an HSC70 functional inhibitor that competitively binds to the ATP-binding pocket of $\operatorname{HSC70}(34,35)$. As shown in Fig. 6C-D, the yield of MBP-positive OLs was significantly decreased after VE treatment, which also attenuated the effect of $\mathrm{ML}$ in promoting $\mathrm{OL}$ production. Furthermore, we determined the transcriptional activity of Olig2 by measuring the mRNA expression level of the Olig2 target gene SOX10 in response to Olig2 ${ }^{\mathrm{S} 147 \mathrm{~A}} \mathrm{mRNA}$ transfection and ML and/or VE treatment (Fig. 6E). ML significantly upregulated SOX10 expression in response to Olig2 ${ }^{\text {S147A }}$ mRNA, while VE treatment attenuated this SOX10 induction and the promoting effect of ML on SOX10 upregulation. Taken together, these results indicated that these two HSP70-modulating compounds might regulate OL generation by affecting the transcriptional activity of Olig2 $2^{\text {S147A }}$.

\section{Discussion}

smRNA-driven differentiation strategies for generating hiPSCs or hiPSC-derived specific cell types have been well documented in recent decades $(10,11,36)$. In this study, smRNA encoding a TF was introduced into cells through nongenome-integrating viruses. This approach not only improved the expression level of TF but also prevented the risk of tumorigenicity caused by genome integration. Here, we first report a highly efficient smRNA-based differentiation strategy for generating human OL cells from hiPSC-derived NPCs. We show that repeated induction of Olig2 smRNA with phosphorylation site modification significantly enhanced the efficiency of OL generation from NPCs derived from hiPSCs.

To the best of our knowledge, although some TFs or TF cocktails have been identified as key factors that depend on integration of retroviral vectors in $\mathrm{OL}$ development $(5,14,16-18), \mathrm{OL}$ fate conversion through a single-TF Olig2 activation had not been well achieved. smRNA-based gene delivery has been shown to be highly efficient and safe for cell fate reprogramming (37-40). A major advantage of the smRNA-based single Olig2 described in this article is the ability to design and modify the mRNA translation sequence to promote protein expression and transcriptional activity. It is crucial to regulate the developmental fate switch of NPCs from MN genesis to OL genesis to generate large numbers of functional OLs from hiPSCs. Li et al. reported that the TF Olig2 participates in this MN-OL fate switching and is dephosphorylated at the onset of OL genesis. Replacing serine 147 with an alanine residue (S147A) had been shown to abolish MN production without preventing OL production in transgenic mice, chicks, or cultured P19 cells (23). It seems that by controlling posttranslational modifications of the Olig2 protein, such as phosphorylation, the function of Olig2 in promoting $\mathrm{MN}$ generation in the early neurogenesis stage can be eliminated (21). Indeed, our results confirmed that Olig2 smRNA with a phosphorylation site modification downregulated the expression of the MN-related genes NGN2 and HB9 and enabled higher expression of Olig2, and $\mathrm{O}^{+}$functional $\mathrm{OL}$ generation. These findings are consistent with those reported in other publications. For example, Xue et al. reported that phosphorylation site modification in proneural 
Atoh1 and Ngn2 effectively enhanced lineage-specific neuron generation from hiPSCs (36). Proper posttranslational modifications resulted in more precise localization and higher translation activity. Therefore, smRNA-based Olig2 expression is essential for MN-OL fate switching and further establishes a practical strategy for OL differentiation from hiPSCs.

A major limitation of this smRNA-driven strategy is the inability to directly induce OPCs and differentiation of uncommitted hiPSCs. Indeed, according to our data, forced expression of Olig2 smRNA alone did not result in NG2- or PDGFRa-positive cells; in contrast, only neural-committed NPCs were found (data not shown). This outcome was consistent with previous findings showing that the single TF SOX10 alone could not directly induce an OL fate in hiPSCs $(5,41)$. Recently, Chanoumidou et al. published a rapid protocol for the generation of $\mathrm{O}^{+}$cells induced by ectopic expression of TF cocktails, including SOX10, Olig2, and NKX6.2, in human fibroblasts, which further differentiated into $\mathrm{MBP}^{+}$mature OL-like cells within 16 days (42). TF cocktails show a powerful propensity for inducing OL lineage specification. Olig2 smRNA showed limited ability to achieve OL specification from hiPSCs, partially due to the lack of certain partner proteins. It is logical to assume that other essential TF smRNAs can be identified for directly generating OLs in hiPSCs or even human fibroblasts in future studies.

Reprogramming of hiPSCs into OPCs/OLs largely relies on mechanistic insights into regulators that modulate the protein stability and/or transcriptional activity of Olig2. Our proteomic analysis established the first profiles of Olig2-binding proteins in NPC-derived hiPSCs. Olig2 ${ }^{\mathrm{S} 147 \mathrm{~A}}$ and Olig2 ${ }^{\mathrm{WT}}$ showed differential binding affinity to a set of binding partners. For instance, PARP-1 has been identified as a positive modulator of $\mathrm{OL}$ differentiation and remyelination $(31,43-45)$. The higher binding affinity of Olig2 ${ }^{\text {S147A }}$ for PARP-1 compared to that of Olig2 ${ }^{\mathrm{WT}}$ indicated a potential mechanism in the promotion of Olig2 ${ }^{\text {S147A }}$ differentiation ability, but greater details on these molecular events remain to be further studied. Most importantly, in this study, we found that the HSP70 complex might regulate the transcriptional activity of Olig2. We show, for the first time, that the HSP70 complex is a functional Olig2binding partner and that the HSP70 agonist ML promotes OL generation. The HSP70 complex is widely known as a chaperone that supports the folding of diverse target proteins $(46,47)$. Several studies have shown that constitutively expressed HSP70 is required for the optimal expression of MBP during OL differentiation (48). Our study demonstrated a novel function of the HSP70 complex in promoting the transcriptional activity of Olig2 and its function in OL lineage specification. Our findings open up an entirely new way of perceiving and understanding the structural basis of the Olig2/HSP70 interaction to optimize OL production from NPC-derived hiPSCs. In summary, direct conversion of hiPSCs into OLs by repeated administration of Olig2 smRNA is a rapid, efficient, and inexpensive alternative for generating human OLs for cell-based therapy, disease modeling and identification of remyelination-promoting smallmolecule and peptide compounds.

\section{Conclusion}


Highly efficient hiPSCs differentiation strategies are critical for the application of hiPSC technology in regenerative medicine. Traditional methods based on chemical compounds and viruses are relatively slow, variable, or unsafe. In this study, a highly efficient strategy was established based on using synthetic mRNA coding single transcription factors Olig2 with dephosphorylation modification to drive rapid hiPSC differentiation to functional oligodendrocytes. Furthermore, the HSP70 complex was identified as a positive modulator of this oligodendrocyte induction process. These discoveries will facilitate the applications of hiPSC-derived oligodendrocytes in demyelination's disease modeling and therapy, and guide the development of robust methods for generating various lineage-specific progenies from hiPSCs.

\section{Abbreviations}

smRNA: synthetic modified messenger RNA; OL: oligodendrocyte; OPC: OL progenitor cell; HSP70: heat shock protein 70; hiPSC: human pluripotent stem cell; MN: motor neuron;

\section{Declarations}

\section{Availability of data and materials}

The datasets used and/or analyzed during the current study are available from the corresponding author on reasonable request.

\section{Consent for publication}

Not applicable

\section{Acknowledgements}

We thank Dr. Jiekai Chen from Guangzhou Institutes of Biomedicine and Health Chinese Academy of Science, China) for kindly providing urine-derived hiPSC cell line.

\section{Funding}

This work was supported by the financial support from the Shenzhen Science and Technology Program (Grant No. KQTD20190929173853397), National Natural Science Foundation of China (Grant Nos. 81772449 and 81971081), the open project of State Key Laboratory of Respiratory Disease (No. SKLRDOP-201905), and the project of "Dengfeng Plan" High-level Hospital Construction Opening Project of Foshan Fourth People's Hospital (No. FSSYKF-2020010).

\section{Contributions}

J.X., Y.C. and W.D. designed experiments and interpreted data. J.X., R.W. carried out most of experiments with technical assistance from J.Z., Y.Z., F.H, L.S, and Y.X. conducted the initial cell transplantation 
experiments. Y.C. and W.D. provided critical input to the overall research direction. J.X. and W.D. wrote the paper with input from all co-authors. All authors read and approved the final manuscript

\section{Competing interests}

The authors declare no competing interests.

\section{Ethical approval}

The animal study protocol was approved by the Sun Yat-sen University Animal Use and Care Committee (SYSU-IACUC-2021-000319).

\section{References}

1. Cayre M, Falque M, Mercier O, Magalon K, Durbec P. Myelin Repair: From Animal Models to Humans. Front Cell Neurosci. 2021;15:604865.

2. Goldman SA. Stem and Progenitor Cell-Based Therapy of the Central Nervous System: Hopes, Hype, and Wishful Thinking. Cell Stem Cell. 2016;18(2):174-88.

3. Goldman SA, Nedergaard M, Windrem MS. Glial progenitor cell-based treatment and modeling of neurological disease. Science. 2012;338(6106):491-5.

4. Goldman S. Stem and progenitor cell-based therapy of the human central nervous system. Nat Biotechnol. 2005;23(7):862-71.

5. Garcia-Leon JA, Kumar M, Boon R, Chau D, One J, Wolfs E, et al. SOX10 Single Transcription FactorBased Fast and Efficient Generation of Oligodendrocytes from Human Pluripotent Stem Cells. Stem Cell Reports. 2018;10(2):655-72.

6. Rhoads RE. Synthetic mRNA: Production, Introduction into Cells, and Physiological Consequences. Methods Mol Biol. 2016;1428:3-27.

7. Balmayor ER. Synthetic mRNA - emerging new class of drug for tissue regeneration. Curr Opin Biotechnol. 2021;74:8-14.

8. Grudzien-Nogalska E, Kowalska J, Su W, Kuhn AN, Slepenkov SV, Darzynkiewicz E, et al. Synthetic mRNAs with superior translation and stability properties. Methods Mol Biol. 2013;969:55-72.

9. Anderson BR, Muramatsu H, Nallagatla SR, Bevilacqua PC, Sansing LH, Weissman D, et al. Incorporation of pseudouridine into mRNA enhances translation by diminishing PKR activation. Nucleic Acids Res. 2010;38(17):5884-92.

10. Mandal PK, Rossi DJ. Reprogramming human fibroblasts to pluripotency using modified mRNA. Nat Protoc. 2013;8(3):568-82.

11. Warren L, Manos PD, Ahfeldt T, Loh YH, Li H, Lau F, et al. Highly efficient reprogramming to pluripotency and directed differentiation of human cells with synthetic modified mRNA. Cell Stem Cell. 2010;7(5):618-30. 
12. Hiratsuka K, Monkawa T, Akiyama T, Nakatake Y, Oda M, Goparaju SK, et al. Induction of human pluripotent stem cells into kidney tissues by synthetic mRNAs encoding transcription factors. Sci Rep. 2019;9(1):913.

13. Goparaju SK, Kohda K, Ibata K, Soma A, Nakatake Y, Akiyama T, et al. Rapid differentiation of human pluripotent stem cells into functional neurons by mRNAs encoding transcription factors. Sci Rep. 2017;7:42367.

14. Biswas S, Chung SH, Jiang P, Dehghan S, Deng W. Development of glial restricted human neural stem cells for oligodendrocyte differentiation in vitro and in vivo. Sci Rep. 2019;9(1):9013.

15. Hu BY, Du ZW, Zhang SC. Differentiation of human oligodendrocytes from pluripotent stem cells. Nat Protoc. 2009;4(11):1614-22.

16. Wu L, Liu C, Chang DY, Zhan R, Zhao M, Lam SM, et al. The attenuation of diabetic nephropathy by annexin A1 via regulation of lipid metabolism through AMPK/PPARalpha/CPT1b pathway. Diabetes. 2021.

17. Wang J, Pol SU, Haberman AK, Wang C, O'Bara MA, Sim FJ. Transcription factor induction of human oligodendrocyte progenitor fate and differentiation. Proc Natl Acad Sci U S A. 2014;111(28):E288594.

18. Ehrlich M, Mozafari S, Glatza M, Starost L, Velychko S, Hallmann A-L, et al. Rapid and efficient generation of oligodendrocytes from human induced pluripotent stem cells using transcription factors. Proceedings of the National Academy of Sciences. 2017;114(11):E2243-E52.

19. Namchaiw P, Wen H, Mayrhofer F, Chechneva O, Biswas S, Deng W. Temporal and partial inhibition of GLI1 in neural stem cells (NSCs) results in the early maturation of NSC derived oligodendrocytes in vitro. Stem Cell Res Ther. 2019;10(1):272.

20. Liu Z, Yan M, Liang Y, Liu M, Zhang K, Shao D, et al. Nucleoporin Seh1 Interacts with Olig2/Brd7 to Promote Oligodendrocyte Differentiation and Myelination. Neuron. 2019;102(3):587-601.

21. Du ZW, Li XJ, Nguyen GD, Zhang SC. Induced expression of Olig2 is sufficient for oligodendrocyte specification but not for motoneuron specification and astrocyte repression. Mol Cell Neurosci. 2006;33(4):371-80.

22. Sher F, Amor S, Gerritsen W, Baker D, Jackson SL, Boddeke E, et al. Intraventricularly injected Olig2NSCs attenuate established relapsing-remitting EAE in mice. Cell Transplant. 2012;21(9):1883-97.

23. Li H, de Faria JP, Andrew P, Nitarska J, Richardson WD. Phosphorylation regulates OLIG2 cofactor choice and the motor neuron-oligodendrocyte fate switch. Neuron. 2011;69(5):918-29.

24. Li W, Sun W, Zhang Y, Wei W, Ambasudhan R, Xia P, et al. Rapid induction and long-term self-renewal of primitive neural precursors from human embryonic stem cells by small molecule inhibitors. Proc Natl Acad Sci U S A. 2011;108(20):8299-304.

25. Borrow P, Martinez-Sobrido L, de la Torre JC. Inhibition of the Type I Interferon Antiviral Response During Arenavirus Infection. Viruses-Basel. 2010;2(11):2443-80.

26. Liu ZJ, Hu XM, Cai J, Liu B, Peng XZ, Wegner M, et al. Induction of oligodendrocyte differentiation by Olig2 and Sox10: Evidence for reciprocal interactions and dosage-dependent mechanisms. Dev Biol. 
2007;302(2):683-93.

27. Zhu Q, Zhao XF, Zheng K, Li H, Huang H, Zhang ZY, et al. Genetic evidence that Nkx2.2 and Pdgfra are major determinants of the timing of oligodendrocyte differentiation in the developing CNS. Development. 2014;141(3):548-55.

28. Butts BD, Houde $\mathrm{C}$, Mehmet $\mathrm{H}$. Maturation-dependent sensitivity of oligodendrocyte lineage cells to apoptosis: implications for normal development and disease. Cell Death Differ. 2008;15(7):1178-86.

29. Boggs JM. Myelin basic protein: a multifunctional protein. Cell Mol Life Sci. 2006;63(17):1945-61.

30. Najm FJ, Lager AM, Zaremba A, Wyatt K, Caprariello AV, Factor DC, et al. Transcription factormediated reprogramming of fibroblasts to expandable, myelinogenic oligodendrocyte progenitor cells. Nat Biotechnol. 2013;31(5):426-33.

31. Wang Y, Zhang YH, Zhang S, Kim BK, Hull VL, Xu J, et al. PARP1-mediated PARylation activity is essential for oligodendroglial differentiation and CNS myelination. Cell Reports. 2021;37(1).

32. Bonam SR, Ruff M, Muller S. HSPA8/HSC70 in Immune Disorders: A Molecular Rheostat that Adjusts Chaperone-Mediated Autophagy Substrates. Cells. 2019;8(8).

33. Calamini B, Silva MC, Madoux F, Hutt DM, Khanna S, Chalfant MA, et al. Small-molecule proteostasis regulators for protein conformational diseases. Nat Chem Biol. 2011;8(2):185-96.

34. Stricher F, Macri C, Ruff M, Muller S. HSPA8/HSC70 chaperone protein: structure, function, and chemical targeting. Autophagy. 2013;9(12):1937-54.

35. Yang X, Tohda C. Heat Shock Cognate 70 Inhibitor, VER-155008, Reduces Memory Deficits and Axonal Degeneration in a Mouse Model of Alzheimer's Disease. Front Pharmacol. 2018;9:48.

36. Xue Y, Zhan X, Sun S, Karuppagounder SS, Xia S, Dawson VL, et al. Synthetic mRNAs Drive Highly Efficient iPS Cell Differentiation to Dopaminergic Neurons. Stem Cells Transl Med. 2019;8(2):11223.

37. Li M, Sancho-Martinez I, Izpisua Belmonte JC. Cell fate conversion by mRNA. Stem Cell Res Ther. 2011;2(1):5.

38. Alvarez-Palomo AB, Requena-Osete J, Delgado-Morales R, Moreno-Manzano V, Grau-Bove C, Tejera $A M$, et al. A synthetic mRNA cell reprogramming method using CYCLIN D1 promotes DNA repair, generating improved genetically stable human induced pluripotent stem cells. Stem Cells. 2021;39(7):866-81.

39. Wang AYL. Application of Modified mRNA in Somatic Reprogramming to Pluripotency and Directed Conversion of Cell Fate. Int J Mol Sci. 2021;22(15).

40. Warren L, Lin C. mRNA-Based Genetic Reprogramming. Mol Ther. 2019;27(4):729-34.

41. Garcia-Leon JA, Garcia-Diaz B, Eggermont K, Caceres-Palomo L, Neyrinck K, Madeiro da Costa R, et al. Generation of oligodendrocytes and establishment of an all-human myelinating platform from human pluripotent stem cells. Nat Protoc. 2020;15(11):3716-44.

42. Chanoumidou K, Hernandez-Rodriguez B, Windener F, Thomas C, Stehling M, Mozafari S, et al. Onestep Reprogramming of Human Fibroblasts into Oligodendrocyte-like Cells by SOX10, OLIG2, and 
NKX6.2. Stem Cell Reports. 2021;16(4):771-83.

43. Plane JM, Grossenbacher SK, Deng W. PARP-1 deletion promotes subventricular zone neural stem cells toward a glial fate. J Neurosci Res. 2012;90(8):1489-506.

44. Charriaut-Marlangue C, Leconte C, Csaba Z, Chafa L, Pansiot J, Talatizi M, et al. Sex differences in the effects of PARP inhibition on microglial phenotypes following neonatal stroke. Brain Behav Immun. 2018;73:375-89.

45. Brochier $\mathrm{C}$, Jones $\mathrm{JI}$, Willis DE, Langley B. Poly(ADP-ribose) polymerase 1 is a novel target to promote axonal regeneration. Proc Natl Acad Sci U S A. 2015;112(49):15220-5.

46. Kumada K, Fuse N, Tamura T, Okamori C, Kurata S. HSP70/DNAJA3 chaperone/cochaperone regulates NF-kappaB activity in immune responses. Biochem Biophys Res Commun. 2019;513(4):947-51.

47. Fernandez-Fernandez MR, Valpuesta JM. Hsp70 chaperone: a master player in protein homeostasis. F1000Res. 2018;7.

48. Aquino DA, Peng D, Lopez C, Farooq M. The constitutive heat shock protein-70 is required for optimal expression of myelin basic protein during differentiation of oligodendrocytes. Neurochem Res. 1998;23(3):413-20.

\section{Figures}

\section{Figure 1}

\section{smRNA coding Olig2 with phosphosite modification show enhanced protein expression and oligodendroglial lineage differentiation ability.}

A Diagram of mRNA coding wild-type and phosphosite-modified Olig2 (Olig2 WT, Olig2 S147A. B Analysis of smRNA transfection efficiency in hiPSC-derived NPCs using 10ng, 50ng, 100ng, 150ng, and 300ng eGFP mRNA at $24 \mathrm{~h}$ post transfection. The representative overlay histogram was presented and the transfection efficiency of NPCs treated with 300ng eGFP mRNA was nearly 95\% $(n=3)$. C NPCs were transfected with GFP, wild-type Olig2 or mutant Olig2 (Flag-tagged) mRNA as indicated. 24h after transfection, fluorescence microscopic images showed high $\mathrm{GFP}^{+}$or Flag ${ }^{+}$cells (DAPI: nuclei; scale bar, $200 \mu \mathrm{m})$. D NPCs received mRNA transfection, and total proteins were harvested at indicated time points for Olig2 western blot. Protein fold expression normalized to tubulin are shown below each lane. E NPCs received three $(\times 3)$ or six $(\times 6)$ daily mRNA transfection. Total RNA was isolated for SOX10 quantitative real-time polymerase chain reaction (qPCR) at d12. F qPCR analysis for the mRNA expression level of SOX10 at d12. Olig2 ${ }^{\mathrm{S147A}}$ more potently induce their downstream targets than their wild-type forms $(n=3$, ns $p>0.05,{ }^{* \star} p<0.01$, by unpaired student $t$ test). 


\section{Figure 2}

\section{A highly efficient strategy for generating smRNA-induced oligodendroglial lineage cells from hiPSC- derived NPCs}

A Diagram of the 6-day mRNA differentiation protocol from hiPSCs. Representative immunofluorescent staining images of human NANOG and SSEA4 (D) proteins in cultured hiPSCs (B), scale bar, $200 \mu \mathrm{m}$. Representative immunofluorescent staining images of human PAX6 and NESTIN (scale bar, 20um) (E) proteins in NPCs (C) derived from hiPSCs (scale bar, 200 $\mu \mathrm{m}$ ). F Representative immunofluorescent staining images of NG2, SOX10, and PDGFRa in mRNA-induced culture at d6 (sacle bar, 50 $\mu \mathrm{m}$ ). Olig2 ${ }^{\mathrm{S} 147 \mathrm{~A}}$ mRNA induced more $\mathrm{NG}^{+} / \mathrm{SOX} 10^{+} / \mathrm{PDGFRa}^{+}$OPC production compared to Olig2 ${ }^{\mathrm{WT}}$ mRNA. G Representative flow cytometry analyses for the expression of NG2 and PDGFRa in mRNA-induced culture at $\mathrm{d} 6$. $\mathbf{H}$ The corresponding quantification of $\mathrm{NG}^{+}$and PDGFRa ${ }^{+}$cells at $\mathrm{d} 6$ in Olig2 ${ }^{\mathrm{WT}}$ or Olig2 ${ }^{\mathrm{S} 147}$ mRNA-induced cultures $(n=3, * * \star p<0.001$, by a two-tailed Student's $t$ test). I The mRNA expression levels of oligodendroglial lineage genes (SOX10) involved in OL differentiation and MN specific maker genes (HB9 and NGN2) at d6 after mRNA transfection $\left(n=3,{ }^{\star \star} \mathrm{p}<0.01,{ }^{\star \star \star} \mathrm{p}<0.001\right.$, by a two-tailed Student's $t$ test).

\section{Figure 3}

\section{Maturation of Olig2 smRNA-induced OPCs into myelinating OLs}

A Representative flow cytometry analyses for the expression of 04 in the Olig2 ${ }^{\mathrm{WT}}$ and Olig2 ${ }^{\mathrm{S} 147 \mathrm{~A}}$ cultures 7, 14 and 21d after smRNA induction. B Quantification of $04^{+}$cells in the Olig2WT and Olig2 $2147 \mathrm{~A}$ cultures 7,14 and $21 \mathrm{~d}$ after smRNA induction $(n=3, * \star * ~ p<0.001$, by two-tailed student $t$ test). $\mathrm{C}$ Immunostaining of induced oligodendrocytes for MBP at d21 after smRNA induction (scale bay, 50 $\mu \mathrm{m}$ ). D Quantification of MBP+ induced oligodendrocytes at day 21 after smRNA induction $(n=3, \star \star ~ p<0.01$, by two-tailed student $t$ test). E The mRNA expression level at d21 of oligodendrocyte differentiation timeline $(n=3, * \star \star ~ p<0.001)$. Olig2 ${ }^{\text {S147A }}$ mRNA-induced oligodendrocytes presented with a branched morphology

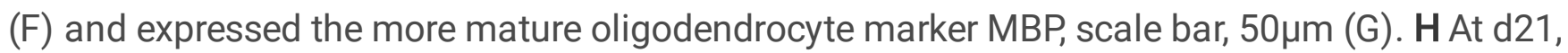
Olig2 ${ }^{\text {S147A }}$ mRNA-induced OLs were further cultured on electrospun nanofibers of $675 \mathrm{~nm}$ diameter, scale bar, $100 \mu \mathrm{m}$. Cultures were grown in mitogen-free media for 2 weeks and immunostained with MBP antibody.

\section{Figure 4}


smRNA-induced OPCs give rise to functional myelin following transplantation in the brain of cuprizoneinduced demyelination mice.

A Diagram showing the corpus callosum transplantation site. B Schematic drawing of the demyelination mouse model and cell transplantation. Female C57BL/ 6 mice were given a diet containing $0.2 \%$ cuprizone for 5 weeks. After inducing demyelination 2 weeks, the purified $04^{+}$OPCs $\left(10^{5}\right)$ were injected into the demyelination mice. C Representative immunofluorescent images for $\mathrm{hN}$ and MBP staining. Transplantation of human OPCs ( $\mathrm{hN}^{+}$cells) into the corpus callosum of mice resulted in extensive generation of MBP+ myelin (green) by human cells and staining positive for the human nuclei marker $\mathrm{hN}$ (red) 8 weeks. scale bar, $20 \mu \mathrm{m}$. D Electron micrographs of myelinated axons exhibiting characteristic compact myelin with alternating major dense $(E$, arrowheads) and intraperiod lines. $(E)$ are higher magnification of The purple box in $D$, scale bar, $200 \mathrm{~nm}$. $\mathbf{F}$ Low magnification electron micrographs showing a portion of corpus callosum from the animals in control, vehicle and OPC transplantation group. myelin sheath (yellow arrowhead, ma), no compact myelin sheath (red, arrowhead, nma). Scale bars, $2 \mu \mathrm{m}$. $\mathbf{G}$ The density of myelinated axons of each group $(n=3)$. Student $t$ test, ${ }^{*} p<0.05$, comparison between vehicle group versus OPC group. $\mathrm{H}$ Mean g-ratio of the control, vehicle group and OPC group. The myelin g-ratio is the ratio of the inner to the outer radius of the myelin sheath for a circular axon cross section. Student $t$ test, ${ }^{\star} p<0.05$ comparison between vehicle group versus OPC group. I Representative images of the corpus callosum region stained with Luxol fast blue after cuprizone and OPC transplantation. scale bars, $200 \mu \mathrm{m}$.

\section{Figure 5}

\section{Proteomic analysis identified proteins differentially binding to 0 lig2 ${ }^{\mathrm{S147A}}$ and $\mathrm{Olig}{ }^{\mathrm{WT}}$.}

A Scheme of the proteomic analysis using NPCs transfected by Olig2 ${ }^{\mathrm{S} 147 \mathrm{~A}}$ and Olig2 ${ }^{\mathrm{WT}}$ mRNA. B Western blotting shows the immunoprecipitation (IP) of Olig2 $2^{\text {S147A }}$ and Olig2 WT proteins by the Flag tag antibody but not mouse $\operatorname{lgG}$. C Differential proteins from Olig2 ${ }^{\mathrm{S147A}}$ and Olig2 ${ }^{\mathrm{WT}}$ were analyzed in the DAVID Bioinformatics Database to identify enriched in myelin sheath pathway (ranked by $-\log 10$ ( $p$ Value)) calculated by the Fisher's exact test. D The Olig2S147A/Olig2 WT binding ratio of HSP70 complex components (HSPA5, HSPA8 and HSPA9) in the myelin sheath pathway.

\section{Figure 6}

The HSP70 complex bind to Olig2 and promotes Olig2-driven mRNA-induced OL differentiation. 


\begin{abstract}
A Diagram of the HSP70 complex and our working model. Two main nuclear components of HSP70 were identified in our study. The HSP70 agonist VER-155008 (VR) and antagonist ML346 (ML) were utilized for inducing and inhibiting HSP70, respectively. B NPCs with Olig2 ${ }^{\mathrm{WT}}$ or Olig2 ${ }^{\mathrm{S} 147 \mathrm{~A}}$ mRNA transfection for 24 hours and nuclear protein were extracted for Flag immunoprecipitation (IP). IP samples were immunoblotted for components of the HSP70 complex. C VR and ML were used along with Olig2 ${ }^{\text {S147A }}$ mRNA during OL differentiation. MBP positive OLs were determined at day 7 of differentiation. D Quantification for the expression of MBP positive cells $(n=3, * * p<0.01, * * p<0.001$, by the Tukey multiple comparison tests). E VR and ML were tested with two daily transfections of Olig2 ${ }^{\text {S147A }} \mathrm{mRNAs}$ in NPCs. At 24 hours after transfection, SOX10 mRNA expression was quantified by $q P C R(n=3, * p<0.05$, by the Tukey multiple comparison tests)

\section{Supplementary Files}

This is a list of supplementary files associated with this preprint. Click to download.

- supplementalmethodsandmaterialstheranostic.docx 